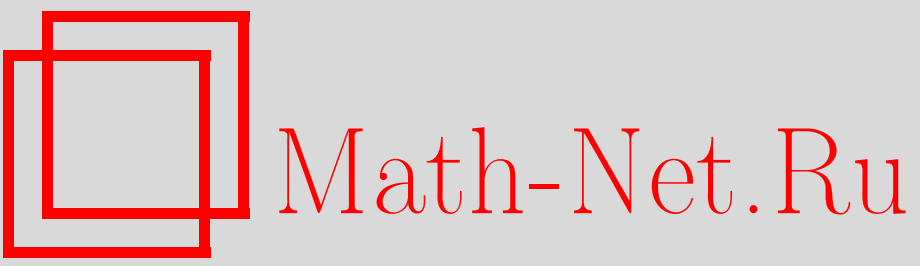

Н. Р. Садыков, Дополнительное искривление траекторий спиновых частиц, пропорциональное кручению траектории, ТМФ, 2005, том 144, номер 3, 555-563

DOI: https://doi.org/10.4213/tmf1876

Использование Общероссийского математического портала Math-Net.Ru подразумевает, что вы прочитали и согласны с пользовательским соглашением

http://www . mathnet.ru/rus/agreement

Параметры загрузки:

IP : 54.92 .164 .108

26 апреля 2023 г., 16:22:31 
ТЕОРЕТИЧЕСКАЯ

И МАТЕМАТИЧЕСКАЯ

ФИЗИКА

Том 144, № 3

сентябрь, 2005

(C) 2005 г.

Н. Р. Садыков*

\section{ДОПОЛНИТЕЛЬНОЕ ИСКРИВЛЕНИЕ ТРАЕКТОРИЙ СПИНОВЫХ ЧАСТИЦ, ПРОПОРЦИОНАЛЬНОЕ КРУЧЕНИЮ ТРАЕКТОРИИ}

В приближении геометрической оптики предсказан эффект дополнительного искривления траекторий частиц (аналог обратного оптического эффекта Магнуса). Эффект определяется поляризацией (спиральностью) и кручением траекторий частиц. Установлена связь рассмотренного эффекта с фазой Берри. Эффект является следствием закона сохранения момента импульса частиц. Показано, что для ультрахолодных нейтронов эффект должен привести к отклонению от закона зеркального отражения.

Ключевые слова: поляризация, оптический эффект Магнуса, спиральность, нейтроновод, электростатическая линза, фаза Берри.

\section{1. ВВЕДЕНИЕ}

В настоящее время в различных областях физики исследуются такие явления, связанные с поляризацией частиц, как спиновые эффекты в физике высоких энергий [1] и при делении ядер [2], эффекты нарушения пространственной четности при радиационном захвате нейтронов [3] и при упругом взаимодействии с ядрами [4], поляризационные эфффекты в оптике [5]- [14].

При изучении поляризационных эффектов в оптике фотонов взаимно дополняют друг друга два подхода - волновой и геометрический (корпускулярный). Оба эти подхода позволяют описать ряд поляризационных (спиновых) эффектов для фотонов: оптический эффект Магнуса [5], [6], [15]-[17] и обратный оптический эффект Магнуса [6], [18] (или эффекты Федорова), влияние величины кручения многомодового световода на величину угла поворота спекл-картины на выходе из волокна [19], врашение спекл-картины в поглощающем световоде [15], [20], [21] и др. Поляризационные эффекты в оптике определяются знаком циркулярной поляризации (спиральностью фотона).

В последнее время часть предсказанных для фотонов эффектов удалось обобщить на спиновые частицы с полуцелым спином и ненулевой массой: в рамках волнового подхо-

\footnotetext{
* Российский федеральный ядерный центр, Всероссийский научно-исследовательский институт технической физики им. акад. И.Е. Забабахина, Снежинск, Челябинская обл., Россия. Е-mail: n.r.sadykov@vniitf.ru
} 
да отметим аналог оптического эффекта Магнуса [22], в рамках корпускулярного подхода - эффект дополнительного искривления траекторий спиновых частиц в поглощающих средах [23].

В работе [22] для спиновых частиц в приближении геометрической оптики (условие квазиклассичности) показано, что происходит дополнительное кручение траектории, пропорциональное кривизне траектории: при движении частищы по круговой траектории у волнового вектора появляется поперечная к плоскости траектории компонента. Наличие волнового вектора позволяет записать эйконал для частицы. Уравнение траектории спиновой частицы получено с помощью вариационного метода, примененного к эйконалу частицы.

В данной работе мы покажем, что при соответствующем преобразовании операторов импульса и динамических переменных в уравнении Дирака в ортогональной (геликоидальной) системе координат из волнового уравнения второго порядка следует, что продольная компонента волнового вектора содержит дополнительное слагаемое (производную фазы Берри по натуральному параметру [7], [24]). Величина этого слагаемого пропорциональна кручению траектории и определяется поляризацией частицы. Полученные результаты по аналогии с оптическим эффектом Магнуса для частиц позволяет получить с помощью эйконала частицы уравнение траектории, исходя из вариационного принципа. На основе анализа полученного уравнения предсказан эффект дополнительного искривления траектории частиц (этот эффект является обратным по отношению к аналогу оптического эффекта Магнуса). Величина эффекта определяется поляризацией и пропорциональна кручению траектории частицы. В искривленной (тороидальной) системе координат используемый в работе подход позволяет обобшить результаты работы [22]: дополнительное к волновому вектору слагаемое, пропорциональное кривизне траектории, строго параллельно бинормали к кривой траектории, что, в свою очередь, приводит к смешению радиуса кривизны траектории вдоль бинормали и не меняет абсолютного значения вектора кривизны.

\section{2. АНАЛОГ ОПТИЧЕСКОГО ЭФФЕКТА МАГНУСА ДЛЯ ЧАСТИЦЫ СО СПИНОМ $s=1 / 2$}

В этом разделе воспользуемся тороидальной системой координат (см. приложения в работах $[22],[23])$ для вывода уравнения траектории, позволяюшего описать аналог оптического эффекта Магнуса для спиновой частицы $s=1 / 2$. Следуя работе [22], введем коэффициенты Ламэ для ортогональной тороидальной системы координат:

$$
h_{1}=1, \quad h_{2}=1, \quad h_{3}=1+\frac{x}{R_{0}},
$$

где $R_{0}$ - радиус тора, $z$ - натуральный параметр, отсчитываемый вдоль окружности радиуса $R_{0}, x$ и $y$ - координаты, базисные векторы которых ортогональны друг другу и перпендикулярны траектории частицы, причем $x$ отсчитьвается вдоль радиуса кривизны $\vec{R}_{0}$ траектории частицы. С точностью до величин порядка $1 / R_{0}$ тороидальная система координат в окрестности точки $x=y=z=0$ совпадает с декартовой. 
Для вывода уравнения траектории в волновом уравнении второго порядка выразим оператор 4-импульса $\widehat{P}=\left(\widehat{P}_{0},-\overrightarrow{\widehat{\mathbf{P}}}\right)$ и динамические переменные $\nu^{\mu}$ через параметры тороидальной системы координат и учтем преобразование волновой функции частицы в результате вращения при перемешении частицы на расстояние $\delta z \rightarrow 0$ по искривленной траектории. Из дальнейшего анализа полученного волнового уравнения получим, что волновой вектор содержит бинормальную компоненту, пропорциональную кривизне траектории частищы и определяемую поляризацией частицы. Записав действие по аналогии с работой [17], получим уравнение траектории частицы.

В квазиклассическом приближении для случая, когда состояние частицы характеризуется определенными значениями энергии, импульса и проекции спина на импульс, отождествим операторы $\hat{\nu}^{n}$ и $\widehat{P}_{n}$ с базисными векторами $\overrightarrow{\mathbf{e}}^{n}$ и $\overrightarrow{\mathbf{e}}_{n}$, имея в виду, что $\hat{\nu}^{n}$ и $\widehat{P}_{n}$ являются соответствующими компонентами векторных операторов в криволинейной системе координат ([25], с. 398):

$$
\hat{\nu}^{n}=\overrightarrow{\mathbf{e}}^{n} \overrightarrow{\hat{\boldsymbol{\nu}}}, \quad \widehat{P}_{n}=\overrightarrow{\mathbf{e}}_{n} \overrightarrow{\widehat{\mathbf{P}}}, \quad \overrightarrow{\hat{\nu}}=\left(\hat{\nu}_{x}, \hat{\nu}_{y}, \hat{\nu}_{z}\right), \quad \overrightarrow{\widehat{\mathbf{P}}}=\left(\widehat{P}_{x}, \widehat{P}_{y}, \widehat{P}_{z}\right),
$$

где $n=1,2,3$ (в дальнейшем латинские индексы принимают значения $1,2,3$, а греческие - значения $0,1,2,3)$. Операторы $\hat{\nu}^{n}$ из (1) удовлетворяют следуюшим соотношениям $([26]$, с. 100$)$ :

$$
\begin{array}{clrl}
\frac{1}{2}\left(\hat{\nu}^{n} \hat{\nu}^{m}+\hat{\nu}^{m} \hat{\nu}^{n}\right) & =g^{n m}, & \frac{1}{2}\left(\hat{\nu}^{n} \hat{\nu}^{m}-\hat{\nu}^{m} \hat{\nu}^{n}\right) & =-i \varepsilon^{n m k} \widehat{\Sigma}_{k}, \\
\frac{1}{2}\left(\widehat{\Sigma}^{n} \widehat{\Sigma}^{m}-\widehat{\Sigma}^{m} \widehat{\Sigma}^{n}\right) & =i \varepsilon^{n m k} \widehat{\Sigma}_{k}, & \frac{1}{2}\left(\widehat{\Sigma}^{n} \hat{\nu}^{m}-\widehat{\Sigma}^{m} \hat{\nu}^{n}\right) & =i \varepsilon^{n m k} \widehat{\Sigma}_{k},
\end{array}
$$

где $\varepsilon^{n m k}=e^{n m k} / \sqrt{g}, e^{n m k}-$ кососимметричный тензор, $g$ - определитель метрического тензора трехмерной системы координат.

При выполнении условий (1) для ковариантной производной имеют место равенства $([25]$, c. 398$)$

$$
\widehat{\nabla}_{n} \hat{\nu}^{n}=\hat{\nu}^{n} \widehat{\nabla}_{n}, \quad(\overrightarrow{\hat{\mathbf{P}}} \overrightarrow{\hat{\boldsymbol{\nu}}})^{2}=-\hbar^{2} \Delta-i \hbar^{2}(\overrightarrow{\widehat{\nabla}} \ln \sqrt{g} \times \overrightarrow{\widehat{\mathbf{\Sigma}}}) \overrightarrow{\hat{\nabla}}
$$

где $\widehat{\nabla}_{n} \overrightarrow{\mathbf{e}}^{m}=0 ; \widehat{\nabla}_{n} \hat{\nu}^{n}=g^{-1 / 2} \partial\left(\sqrt{g} \hat{\nu}^{n}\right) / \partial x^{n} ; \Delta-$ лапласиан.

Теперь учтем преобразование волновой функции при врашении тороидальной системы координат. При движении спиновой частицы $s=1 / 2$ по круговой траектории с радиусом кривизны $R_{0}=1 / \tau_{0}$, где $\overrightarrow{\boldsymbol{\tau}}_{0}=\overrightarrow{\mathbf{R}}_{0} / R_{0}^{2}$ - кривизна траектории, тороидальная система координат врашается относительно неподвижной системы координат на угол $\delta \vec{\Theta}=\vec{\eta} \delta z$, ге $\overrightarrow{\boldsymbol{\eta}}=\overrightarrow{\boldsymbol{\tau}}_{0} \times \overrightarrow{\mathbf{l}}, \overrightarrow{\mathbf{l}}-$ касательный к траектории частицы единичный вектор, $\delta z$ - отсчитьваемая вдоль траектории частицы элементарная длина (натуральный параметр) траектории. При малом повороте биспиноры $\xi$ и $\eta$ преобразуются в соответствии со следующими формулами ([27], с. 81):

$$
\Psi=\left(\frac{\xi}{\eta}\right), \quad \Psi^{\prime}=\left(\frac{\xi^{\prime}}{\eta^{\prime}}\right)=\left(1+i \frac{(\overrightarrow{\hat{\Sigma}} \delta \overrightarrow{\boldsymbol{\Theta}})}{2}\right) \Psi, \quad \overrightarrow{\widehat{\Sigma}}=\left(\begin{array}{cc}
\overrightarrow{\hat{\boldsymbol{\sigma}}} & 0 \\
0 & \overrightarrow{\hat{\boldsymbol{\sigma}}}
\end{array}\right)
$$


При движении частицы по искривленной траектории изменение направления поляризации происходит также за счет прецессии Томаса [28]. В работах [22], [23] было показано, что влияние врашения поляризации частицы на интересуюший нас эффект не является сушественным.

С учетом соотношений (П.1.1), (П.1.5) и равенства $\vec{\nabla} \ln \sqrt{g}=\vec{\tau}_{0}$, справедливого в тороидальной системе координат, получим

$$
\left[(\overrightarrow{\hat{\mathbf{P}}} \overrightarrow{\hat{\boldsymbol{\nu}}})^{2} \Psi^{\prime}\right]_{\delta z=0}=(\overrightarrow{\hat{\mathbf{P}}} \overrightarrow{\hat{\boldsymbol{\nu}}})^{2} \Psi+\hbar(\overrightarrow{\mathbf{l}} \overrightarrow{\mathbf{P}})(\overrightarrow{\boldsymbol{\eta}} \overrightarrow{\hat{\boldsymbol{\Sigma}}}) \Psi=\left[-\hbar^{2} \Delta-i \hbar^{2}\left(\overrightarrow{\boldsymbol{\tau}}_{0} \times \overrightarrow{\hat{\mathbf{\Sigma}}}\right)_{\perp} \overrightarrow{\hat{\boldsymbol{\nabla}}}_{\perp}\right] \Psi .
$$

где знак “ $\perp$ ” означает перпендикулярную к вектору $\overrightarrow{\mathbf{l}}$ составляющую векторного оператора. При выводе соотношения (5) учтено, что $\overrightarrow{\boldsymbol{\eta}}=\overrightarrow{\boldsymbol{\tau}}_{0} \times \overrightarrow{\mathbf{1}}$. В результате имеем

$$
\Delta \Psi+k_{0}^{2} \Psi+i(\overrightarrow{\mathbf{l}} \overrightarrow{\boldsymbol{\Sigma}})(\overrightarrow{\boldsymbol{\eta}} \overrightarrow{\boldsymbol{\nabla}}) \Psi=0
$$

где $k_{0}^{2}=\left[(\varepsilon-\Phi)^{2} / c^{2}-m^{2} c^{2}\right] / \hbar^{2}, \varepsilon-$ энергия частицы, $\left(\overrightarrow{\boldsymbol{\tau}}_{0} \times \overrightarrow{\boldsymbol{\Sigma}}\right)_{\perp}=(\overrightarrow{\mathbf{l}} \overrightarrow{\boldsymbol{\Sigma}}) \overrightarrow{\boldsymbol{\eta}}$.

Представим решение этого уравнения в виде

$$
\Psi=\Psi_{0} e^{(i \overrightarrow{\boldsymbol{\kappa}} \overrightarrow{\mathbf{r}})} .
$$

Величину $\overrightarrow{\boldsymbol{\kappa}}$ выберем по аналогии с работой [17] так, чтобы функция $\Psi_{0}$ удовлетворяла уравнению (6) без последнего слагаемого. Тогда

$$
\overrightarrow{\boldsymbol{\kappa}}=-\frac{1}{2}(\overrightarrow{\mathbf{l}} \overrightarrow{\mathbf{n}}) \overrightarrow{\boldsymbol{\eta}},
$$

где $\overrightarrow{\widehat{\Sigma}} \Psi_{0}=\overrightarrow{\mathbf{n}} \Psi_{0},|\overrightarrow{\mathbf{n}}|=1$.

Если с учетом выражения для $\overrightarrow{\boldsymbol{\kappa}}$ записать действие в окрестности рассматриваемой точки и приравнять нулю его вариацию, то получим уравнение траектории спиновой частицы с полуцелым спином (подробнее см. [17])

$$
\frac{d \overrightarrow{\mathbf{l}}}{d z}=\overrightarrow{\mathbf{l}} \times\left[\vec{\nabla} \ln k_{0}+\frac{1}{k_{0}} \frac{d \overrightarrow{\boldsymbol{\kappa}}}{d z}\right] \times \overrightarrow{\mathbf{l}},
$$

где $\overrightarrow{\boldsymbol{\kappa}}$ зависит от $z$. Учет второго слагаемого в этом уравнении приводит к изменению направления вектора кривизны $\vec{\tau}=\overrightarrow{\mathbf{R}} / R^{2}$ ( $\vec{R}$ - радиус кривизны $)$ на небольшую величину, при этом

$$
\overrightarrow{\boldsymbol{\tau}}=\left[\overrightarrow{\hat{\nabla}} \ln k_{0}+\frac{1}{k_{0}} \frac{d \overrightarrow{\boldsymbol{\kappa}}}{d z}\right]_{\perp} .
$$

При выводе уравнения предполагалось, что кручение траектории отсутствует, поэтому производная $\partial \overrightarrow{\boldsymbol{\eta}} / \partial z$ не может содержать параллельную вектору $\overrightarrow{\boldsymbol{\tau}}_{0}=\overrightarrow{\hat{\nabla}}_{\perp} \ln k_{0}$ компоненту и, тем самым, изменять абсолютное значение кривизны.

Вектор $\overrightarrow{\boldsymbol{\kappa}}$ изменяется как за счет изменения величины $\overrightarrow{\boldsymbol{\eta}}$, так и за счет изменения величины $(\overrightarrow{\mathbf{l}} \overrightarrow{\mathbf{n}})$. При движении в первом приближении можно считать, что $d \overrightarrow{\mathbf{l}} / d z=\overrightarrow{\boldsymbol{\tau}}_{0}$, а $d \overrightarrow{\mathbf{n}} / d z$ определяется прецессией Томаса [28] (см. также [22], [23]). 


\section{3. АНАЛОГ ОБРАТНОГО ОПТИЧЕСКОГО ЭФФЕКТА МАГНУСА (ЭФФЕКТА ФЕДОРОВА) ДЛЯ ЧАСТИЦЫ СО СПИНОМ $s=1 / 2$}

Рассмотрим движение частицы по траектории с ненулевой кривизной и ненулевым кручением. Для сокрашения выкладок будем пренебрегать в (4) врашением системы координат за счет кривизны траектории. Тогда в формуле для $\Psi^{\prime}$ в соотношениях (4) угол $\delta \overrightarrow{\boldsymbol{\Theta}}$ равен углу вращения системы координат за счет кручения:

$$
\delta \vec{\Theta}=\overrightarrow{\mathbf{l}} \tilde{\eta} \delta z,
$$

где $\tilde{\eta}$ - кручение траектории.

Движение частишы удобно описать в геликоидальной системе координат (П.2.1) (уравнение для фотона см. в работе [6]). Для геликоидальной системы координат имеет место равенство $\sqrt{g}=1$. По аналогии с выводом уравнения (5) с учетом (П.1.10) получим

$$
\left[(\overrightarrow{\hat{\mathbf{P}}} \overrightarrow{\boldsymbol{\nu}}) \Psi^{\prime}\right]_{\delta z=0}=(\overrightarrow{\hat{\mathbf{P}}} \overrightarrow{\boldsymbol{\nu}})^{2} \Psi+\hbar \tilde{\eta}(\overrightarrow{\mathbf{1}} \overrightarrow{\boldsymbol{\Sigma}})(\overrightarrow{\mathbf{1}} \overrightarrow{\hat{\mathbf{P}}}) \Psi .
$$

Исходя из этого уравнения, действуя по аналогии с выводом (6), запишем волновое уравнение

$$
\Delta \Psi+k_{0}^{2} \Psi+i \tilde{\eta}(\overrightarrow{\mathbf{l}} \overrightarrow{\widehat{\Sigma}})(\overrightarrow{\mathbf{l}} \overrightarrow{\boldsymbol{\nabla}}) \Psi=0 .
$$

Представим $\Psi$ в виде

$$
\Psi=\Psi_{1} e^{(i \tilde{\kappa} z)} .
$$

Величину $\tilde{\kappa}$ выберем так, чтобы функция $\Psi_{1}$ удовлетворяла уравнению (13) без последнего слагаемого. В результате с учетом вида функции $\Psi$ получим уравнение, аналогичное уравнению (8),

$$
\overrightarrow{\tilde{\kappa}}=-\frac{1}{2}(\overrightarrow{\mathbf{l}} \overrightarrow{\mathbf{n}}) \hat{\eta} \overrightarrow{\mathbf{l}},
$$

где $k=k_{0}-(\overrightarrow{\mathbf{l}} \overrightarrow{\mathbf{n}}) \tilde{\eta} / 2-$ волновое число, выражение для $k_{0}$ приведено после формулы (6).

Формула (15) определяет величину производной фазы Берри $d \Theta / d z[7],[24]$ по натуральному параметру при движении спиновой частицы по геликоидальной траектории: $d \Theta / d z=-m \tilde{\eta}$. В случае движения спиновой частицы по замкнутому контуру в трехмерном пространстве параметров изменение фазы Берри будет равно телесному углу $\Omega$, под которым виден этот контур из начала координат в пространстве параметров [7]: $\Theta=-m \Omega$. Приведенные формулы были получены Берри из предположения адиабатической инвариантности величины $m=(\overrightarrow{\mathbf{l}} \overrightarrow{\mathbf{s}})$. Результат (15) описывает врашение спина относительно трехгранника Френе, что совпадает с прецессией спина относительно этого трехгранника ([9], с. 8).

Прототипом фазы Берри и Панчаратнама [10] в оптике является фаза Рытова-Владимирского, связанная с поворотом плоскости поляризации, [11], [12]. При этом роль параметра выполняет касательный к траектории единичный вектор. Об экспериментальном наблюдении фазы Рытова-Владимирского сообщалось в [13]. Величине фазы 
Рытова-Владимирского равен также угол поворота спекл-картины на выходе из скрученного многомодового световода (данный факт установлен экспериментально [19] и теоретически [6], [14]).

Если с учетом (15) записать действие в окрестности рассматриваемой точки и приравнять нулю его вариацию, то получим уравнение траектории частицы со спином $s=$ 1/2 (подробнее см. [6]):

$$
\frac{d \overrightarrow{\mathbf{l}}}{d z}=\overrightarrow{\mathbf{l}} \times\left[\vec{\nabla} \ln k+\frac{1}{k_{0}} \frac{\partial \overrightarrow{\boldsymbol{\kappa}}}{\partial z}\right] \times \overrightarrow{\mathbf{l}}
$$

где $k$ определяется выражением, приведенным после формулы (15), и содержит параметр кручения, т.е. отличается от $k_{0}$ в $(9)$. При выводе данного уравнения учтено, что $\overrightarrow{\widehat{\nabla}}(\overrightarrow{\mathbf{l}} \overrightarrow{\mathbf{n}})=\overrightarrow{\mathbf{l}} \partial(\overrightarrow{\mathbf{l}} \overrightarrow{\mathbf{n}}) / d z$. Из (16) следует, что кручение траектории приводит к изменению кривизны по абсолютной величине и не меняет направления вектора кривизны. Из этого уравнения также видно, что вклады кривизны и кручения в траекторию частицы соизмеримы по абсолютной величине при выполнении либо условия $|\partial \ln \tau / \partial \mathbf{s}| \sim \kappa$, либо условия $\tau \sim \kappa$.

\section{4. ДВИЖЕНИЕ УЛЬТРАХОЛОДНЫХ НЕЙТРОНОВ}

Покажем, что рассматриваемый в работе эффект, также как и аналог оптического эффекта Магнуса, проявляется при движении ультрахолодных нейтронов. По аналогии с работой [22] аппроксимируем функциональную зависимость потенциальной энергии вблизи стенки нейтроновода при $x>0$ параболой $U(x)=\left(x^{2} / H^{2}\right) \Delta U$, где $\Delta U \sim$ $10^{-7}$ эВ. При $E \sim \Delta U$ полагая $|\vec{l} \vec{n}| \approx 1, \tilde{\eta}=\mathrm{const}$, из (16) получим уравнение траектории

$$
\frac{d^{2} x}{d z^{2}}=-\frac{2 m \Delta U}{H^{2} \hbar^{2}\left(k_{0}-\tilde{\eta} / 2\right)^{2}} x, \quad \frac{d^{2} y}{d z^{2}} \approx-\frac{1}{2 k_{0}} \frac{\Delta U}{E H^{2}} \frac{\partial x}{\partial z},
$$

при этом $\tau_{0} \approx-\Delta U /(E H)$

Из (16) следует, что $\tau \approx \tau_{0} /\left(k_{0}-\tilde{\eta} / 2\right)$. Соответственно, $\Delta \tau=\left|\tau-\tau_{0}\right| \approx \tilde{\eta} \tau_{0} /\left(2 k_{0}\right)$, т.е. $\Delta \tau / \tilde{\eta}=\tau_{0} /\left(2 k_{0}\right)$. При $E \sim \Delta U, H \sim(1 \div 10) \lambda$ получим $\Delta \tau / \tilde{\eta} \sim\left(10^{-2} \div 10^{-1}\right)$. Видно, что рассматриваемый в работе эффект будет проявляться для ультрахолодных нейтронов, также как и в случае аналога оптического эффекта Магнуса, будет наблюдаться сильное отклонение от законов геометрической оптики.

\section{5. ОБСУЖДЕНИЕ РЕЗУЛЬТАТОВ}

Покажем, что аналог обратного оптического эффекта Магнуса, также как и рассмотренный в работах [22] и [23] эффект, является следствием закона сохранения полного момента импульса.

Из (16) следует, что обобшенньй импульс частицы равен $\overrightarrow{\mathbf{P}}=\hbar k \overrightarrow{\mathbf{l}}$. Пусть $r, \varphi, \tilde{z}-$ координаты цилиндрической системы координат.

Соответственно, $\tilde{z}$-компонента орбитального момента $L_{\tilde{z}}$ при движении частищы по геликоидальной траектории будет равна $L_{\tilde{z}}=\operatorname{Pr} \sin \alpha=\hbar k r^{2} \varphi_{\tilde{z}}^{\prime} \cos \alpha$, где $\operatorname{tg} \alpha=r \varphi_{\tilde{z}}^{\prime}$, 


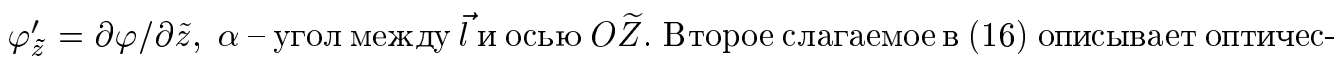
кий эффект Магнуса, который удовлетворяет закону сохранения полного момента импульса [22]. Поэтому вторым слагаемым можно пренебречь, в результате имеем уравнение

$$
\frac{\partial}{\partial z}\left(k r^{2} \varphi_{z}^{\prime}\right)=0
$$

Поскольку $\varphi_{z}^{\prime}=\varphi_{\tilde{z}}^{\prime} \partial \tilde{z} / \partial z=\varphi_{\tilde{z}}^{\prime} \cos \alpha$, то в соответствии с уравнением (18) получим, что $L_{\tilde{z}}=$ const. При $\nu^{2} / c^{2} \ll 1$ прецессией Томаса, как это указывалось в разделе 2 , можно пренебречь, поэтому при движении частицы по геликоидальной траектории проекция вектора $\overrightarrow{\mathbf{n}}$ на ось $O \widetilde{Z}$ также сохраняется. Это означает, что при движении частицы по геликоидальной траектории сохраняется проекция полного момента на ось $O \widetilde{Z}$.

Из полученных результатов следует, что дополнительное искривление траектории спиновой частишы обуславливается наличием дополнительной фазы (11), которая пропорциональна кручению и определяется спиральностью спиновой частишы (геометрическая фаза Ааронова-Анандана [29], являюшаяся обобшением адиабатической фазы Берри). Отсюда следует, что спиральность частицы при наличии кручения траектории изменяет кривизну траектории, а в случае переменной кривизны происходит кручение траектории.

В данной работе обобщены результаты работы [22]. Показано, что дополнительное слагаемое к волновому вектору (8) параллельно бинормали.

Аналог обратного оптического эффекта Магнуса, также как аналог оптического эффекта Магнуса, в области энергий ультрахолодных нейтронов приводит к сильному отклонению от законов геометрической оптики.

ПРИЛОЖЕНИЕ 1

Пусть

$$
\hat{A}=\overrightarrow{\widehat{\mathbf{P}}} \overrightarrow{\hat{\nu}}, \quad \widehat{B}=1+i \frac{1}{2}(\overrightarrow{\boldsymbol{\Sigma}} \delta \overrightarrow{\boldsymbol{\Theta}}),
$$

где $\overrightarrow{\hat{\boldsymbol{\nu}}}$ и $\overrightarrow{\boldsymbol{\Sigma}}$ определены в (2) и (4), $\delta \overrightarrow{\boldsymbol{\Theta}}=\overrightarrow{\boldsymbol{\eta}} \delta z$. Тогда

$$
\begin{aligned}
2\{\hat{A}, \widehat{B}\}_{\delta z=0} \hat{A}+\{\hat{A},\{\hat{A}, \widehat{B}\}\}_{\delta z=0}= & \frac{1}{2} \hbar[(\overrightarrow{\mathbf{l}} \overrightarrow{\boldsymbol{\nu}})(\overrightarrow{\boldsymbol{\eta}} \overrightarrow{\boldsymbol{\Sigma}})(\overrightarrow{\hat{\mathbf{P}}} \overrightarrow{\hat{\boldsymbol{\nu}}})+(\overrightarrow{\widehat{\mathbf{P}}} \overrightarrow{\boldsymbol{\nu}})(\overrightarrow{\mathbf{l}} \overrightarrow{\boldsymbol{\nu}})(\overrightarrow{\boldsymbol{\eta}} \overrightarrow{\widehat{\boldsymbol{\Sigma}}})]- \\
& -\{\overrightarrow{\widehat{\mathbf{P}}} \overrightarrow{\boldsymbol{\nu}}, \delta \overrightarrow{\boldsymbol{\Theta}} \overrightarrow{\hat{\boldsymbol{\nu}}} \times \overrightarrow{\widehat{\mathbf{P}}}\}_{\delta z=0}
\end{aligned}
$$

где

$$
\{\overrightarrow{\hat{\mathbf{P}}} \overrightarrow{\hat{\nu}}, \delta \overrightarrow{\boldsymbol{\Theta}} \overrightarrow{\hat{\boldsymbol{\nu}}} \times \overrightarrow{\widehat{\mathbf{P}}}\}_{\delta z=0}=-i \hbar(\overrightarrow{\mathbf{l}} \overrightarrow{\hat{\nu}})(\overrightarrow{\boldsymbol{\eta}} \times \overrightarrow{\hat{\boldsymbol{\nu}}}) \overrightarrow{\mathbf{P}} .
$$

Выражение в квадратных скобках в правой части (П.1.2) преобразуем к виду

$$
\begin{aligned}
& \frac{1}{2} \hbar[(\overrightarrow{\mathbf{1}} \overrightarrow{\hat{\boldsymbol{\nu}}})(\overrightarrow{\boldsymbol{\eta}} \overrightarrow{\boldsymbol{\Sigma}})(\overrightarrow{\hat{\mathbf{P}}} \overrightarrow{\hat{\boldsymbol{\nu}}})+(\overrightarrow{\hat{\mathbf{P}}} \overrightarrow{\hat{\boldsymbol{\nu}}})(\overrightarrow{\mathbf{1}} \overrightarrow{\hat{\boldsymbol{\nu}}})(\overrightarrow{\boldsymbol{\eta}} \overrightarrow{\boldsymbol{\Sigma}})]=\frac{1}{2} \hbar[((\overrightarrow{\mathbf{1}} \overrightarrow{\hat{\boldsymbol{\nu}}})(\overrightarrow{\hat{\mathbf{P}}} \overrightarrow{\hat{\boldsymbol{\nu}}})+(\overrightarrow{\hat{\mathbf{P}}} \overrightarrow{\hat{\boldsymbol{\nu}}})(\overrightarrow{\mathbf{1}} \overrightarrow{\boldsymbol{\nu}}))(\overrightarrow{\boldsymbol{\eta}} \overrightarrow{\hat{\boldsymbol{\Sigma}}})+ \\
& +(\overrightarrow{\mathbf{l}} \overrightarrow{\boldsymbol{\nu}})\{\overrightarrow{\boldsymbol{\eta}} \overrightarrow{\hat{\Sigma}}, \overrightarrow{\mathbf{P}} \overrightarrow{\hat{\nu}}\}]=\hbar(\overrightarrow{\mathbf{l}} \overrightarrow{\mathbf{P}})(\overrightarrow{\boldsymbol{\eta}} \overrightarrow{\boldsymbol{\Sigma}})+i \hbar(\overrightarrow{\mathbf{l}} \overrightarrow{\boldsymbol{\nu}})(\overrightarrow{\hat{\boldsymbol{\nu}}} \times \overrightarrow{\boldsymbol{\eta}}) \overrightarrow{\mathbf{P}} .
\end{aligned}
$$


С учетом (П.1.2) и (П.1.3) отсюда получим

$$
2\{\hat{A}, \widehat{B}\}_{\delta z=0} \hat{A}+\{\hat{A},\{\hat{A}, \widehat{B}\}\}_{\delta z=0}=\hbar(\overrightarrow{\mathbf{l}} \overrightarrow{\mathbf{P}})(\overrightarrow{\boldsymbol{\eta}} \overrightarrow{\boldsymbol{\Sigma}}),
$$

где операторы $\hat{A}$ и $\widehat{B}$ определены в (П.1.1).

Пусть

$$
\delta \overrightarrow{\boldsymbol{\Theta}}=\overrightarrow{\mathbf{l}} \tilde{\kappa} \delta z .
$$

Отсюда с учетом (П.1.1) по аналогии с выводом (П.1.2) получим

$$
\begin{aligned}
2\{\hat{A}, \widehat{B}\}_{\delta z=0} \hat{A}+\{\hat{A},\{\hat{A}, \widehat{B}\}\}_{\delta z=0}= & \frac{1}{2} \hbar \tilde{\kappa}[(\overrightarrow{\mathbf{l}} \overrightarrow{\boldsymbol{\nu}})(\overrightarrow{\mathbf{l}} \overrightarrow{\boldsymbol{\Sigma}})(\overrightarrow{\hat{\mathbf{P}}} \overrightarrow{\hat{\boldsymbol{\nu}}})+(\overrightarrow{\hat{\mathbf{P}}} \overrightarrow{\hat{\boldsymbol{\nu}}})(\overrightarrow{\mathbf{l}} \overrightarrow{\boldsymbol{\nu}})(\overrightarrow{\mathbf{l}} \overrightarrow{\boldsymbol{\Sigma}})]- \\
& -\{\overrightarrow{\widehat{\mathbf{P}}} \overrightarrow{\hat{\boldsymbol{\nu}}}, \delta \overrightarrow{\boldsymbol{\Theta}} \overrightarrow{\hat{\boldsymbol{\nu}}} \times \overrightarrow{\widehat{\mathbf{P}}}\}_{\delta z=0},
\end{aligned}
$$

где

$$
\{\overrightarrow{\hat{\mathbf{P}}} \overrightarrow{\hat{\nu}}, \delta \overrightarrow{\boldsymbol{\Theta}} \overrightarrow{\hat{\nu}} \times \overrightarrow{\hat{\mathbf{P}}}\}_{\delta z=0}=+i \hbar \tilde{\kappa}(\overrightarrow{\mathbf{l}} \overrightarrow{\hat{\boldsymbol{\nu}}})(\overrightarrow{\hat{\boldsymbol{\nu}}} \times \overrightarrow{\mathbf{l}}) \overrightarrow{\hat{\mathbf{P}}} .
$$

Выражение в квадратных скобках в правой части (П.1.7) преобразуем к виду

$$
\begin{aligned}
& \frac{1}{2} \hbar \tilde{\kappa}[(\overrightarrow{\mathbf{l}} \overrightarrow{\hat{\boldsymbol{\nu}}})(\overrightarrow{\mathbf{l}} \overrightarrow{\boldsymbol{\Sigma}})(\overrightarrow{\hat{\mathbf{P}}} \overrightarrow{\hat{\boldsymbol{\nu}}})+(\overrightarrow{\mathbf{P}} \overrightarrow{\hat{\boldsymbol{\nu}}})(\overrightarrow{\mathbf{l}} \overrightarrow{\hat{\boldsymbol{\nu}}})(\overrightarrow{\mathbf{l}} \overrightarrow{\boldsymbol{\Sigma}})]=\frac{1}{2} \hbar[((\overrightarrow{\mathbf{l}} \overrightarrow{\hat{\boldsymbol{\nu}}})(\overrightarrow{\mathbf{P}} \overrightarrow{\hat{\boldsymbol{\nu}}})+(\overrightarrow{\hat{\mathbf{P}}} \overrightarrow{\hat{\boldsymbol{\nu}}})(\overrightarrow{\mathbf{l}} \overrightarrow{\hat{\boldsymbol{\nu}}}))(\overrightarrow{\mathbf{l}} \overrightarrow{\boldsymbol{\Sigma}})+ \\
& +(\overrightarrow{\mathbf{l}} \overrightarrow{\hat{\nu}})\{\overrightarrow{\mathbf{l}} \overrightarrow{\boldsymbol{\Sigma}}, \overrightarrow{\hat{\mathbf{P}}} \overrightarrow{\hat{\nu}}\}]=\hbar \tilde{\kappa}(\overrightarrow{\mathbf{l}} \overrightarrow{\boldsymbol{\Sigma}})(\overrightarrow{\mathbf{l}} \overrightarrow{\hat{\mathbf{P}}})+i \hbar \tilde{\nu}(\overrightarrow{\mathbf{l}} \overrightarrow{\hat{\nu}})(\overrightarrow{\hat{\boldsymbol{\nu}}} \times \overrightarrow{\mathbf{l}}) \overrightarrow{\hat{\mathbf{P}}} .
\end{aligned}
$$

С учетом (П.1.7), (П.1.8) отсюда получим

$$
2\{\hat{A}, \widehat{B}\}_{\delta z=0} \hat{A}+\{\hat{A},\{\hat{A}, \widehat{B}\}\}_{\delta z=0}=\hbar \tilde{\kappa}(\overrightarrow{\mathbf{l}} \overrightarrow{\boldsymbol{\Sigma}})(\overrightarrow{\mathbf{l}} \overrightarrow{\mathbf{P}}) .
$$

ПРИЛОЖКНИИ 2

Введем криволинейную систему координат

$$
\begin{aligned}
& x^{1}=x \cos \varkappa z+y \sin \varkappa z, \\
& x^{2}=-x \sin \varkappa z+y \cos \varkappa z, \\
& x^{3}=z .
\end{aligned}
$$

Используя известные из курса дифференциальной геометрии формулы для базисных векторов $\overrightarrow{\mathbf{e}}_{\alpha}^{\prime}$ (см. [25]), получим

$$
g_{\alpha \beta}=\left(\begin{array}{ccc}
1 & 0 & \varkappa y \\
0 & 1 & -\varkappa x \\
\varkappa y & -\varkappa x & 1+\varkappa^{2}\left(x^{2}+y^{2}\right)
\end{array}\right),
$$

где для метрического тензора имеет место соотношение $g_{\alpha \beta}=\overrightarrow{\mathbf{e}}_{\alpha}^{\prime} \overrightarrow{\mathbf{e}}_{\beta}^{\prime}$.

Из (П.2.2) видно, что определитель $g$ метрического тензора $g_{\alpha \beta}$ равен единице, а при $x=y=0$ система координат является декартовой. 


\section{Список литературы}

[1] С. М. Трошин, Н.Е. Тюрин. УФН. 1994. Т. 164. С. 1073.

[2] Г. В. Данилян. УФН. 1980. Т. 131. С. 329.

[3] Д. В. Зарецкий, В. К. Сироткин. ЯФ. 1984. Т. 39. С. 585; E. Kolomensky, V. M. Lobashev, A. V. Pirozhkov et al. Phys. Lett. B. 1981. V. 107. P. 272; Yu. G. Abov, P. A. Krupchitssky, Yи. A. Oratovsky. Phys. Lett. 1964. V. 12. Р. 25; В.М. Лобашев, В. Л. Назаренко, Л. Ф. Саенко и др. Письма в ЖЭТФ. 1966. Т. 3. С. 268.

[4] Л.П. Горьков, И.Е. Дзялоиинский. ЖЭТФ. 1974. Т. 67. С. 397; В. П. Алфименков. УФН. 1984. Т. 144. С. 361.

[5] Б. Я. Зельдович, В. С. Либерман. Квантовая электроника. 1990. Т. 17. С. 493; А. В. Дугин, Б. Я. Зельдович, Н. Д. Кундикова, В. С. Либерман. Письма в ЖЭТФ. 1991. Т. 53. С. 186; ЖЭТФ. 1991. Т. 100. С. 1474; А. В. Воляр, С. Н. Лапаева. Письма в ЖТФ. 1992. Т. 18. № 8. С. $53 ;$ 1994. Т. 20. № 8. С. 4; Н. Р. Садыков. Оптика и спектроскопия. 2000. Т. 89 № 2. С. 273 ; Ф. И. Федоров. ДАН СССР. 1955. Т. 105. С. 465; ЖПС. 1977. Т. 27. С. 580.

[6] Н. Р. Садыков. Оптика и спектроскопия. 1998. Т. 84. № 4. С. 300

[7] M. V. Berry. Pros. Roy. Soc. London. Ser. A. 1984. V. 392. P. 45; Sci. American. 1988. V. 259. № 6. P. 26.

[8] Д. Н. Кльикко. УФН. 1993. Т. 163. С. 1.

[9] С.И. Виницкий, В. Л. Дербов, В. М. Дубовик, Б. Д. Марковски, Ю. П. Степановский. УФН. 1990. Т. 160. № 6. С. 1.

[10] S. Pancharatnam. Proc. Indian Acad. Sci. Ser. A. 1956. V. A44. P. 247.

[11] C. М. Рьтов. ДАН СССР. 1938. Т. 18. С. 263.

[12] В. В. Владимирский. ДАН СССР. 1941. Т. 21. С. 222.

[13] A. Tomita, R. Y. Chiao. Phys. Rev. Lett. 1986. V. 57. P. 937

[14] Н. Р. Садыков. Известия высших учебных заведений. Физика. 1999. № 10. С. 58.

[15] Н. Р. Садыков. Квантовая электроника. 1993. Т. 20. С. 1140.

[16] Н. Р. Садыков. Оптика и спектроскопия. 1995. Т. 78. № 2. С. 300.

[17] Н. Р. Садыков. Квантовая электроника. 1992. Т. 19. С. 1021.

[18] Н. Р. Садьков. Квантовая электроника. 1996. Т. 23. С. 277.

[19] И. В. Катаевская, Н. Д. Кундикова. Квантовая электроника. 1995. Т. 22. С. 959.

[20] Н. Д. Кундикова, В. Г. Николаев, Н. Р. Садъков, М. О. Садыкова. Оптика и спектроскопия. 2003. Т. 94. № 4. С. 699.

[21] Н. Р. Садыћков. ДАН. 2003. Т. 389. № 1. С. 38.

[22] Н. Р. Садыков. ТМФ. 2003. Т. 135. № 2. С. 280.

[23] Н. Р. Садыков. ТМФ. 2004. Т. 139. № 3. С. 491.

[24] B. Simon. Phys. Rev. Lett. 1983. V. 51. P. 2167.

[25] Б. А. Дубровин, С. П. Новиков, А. Т. Фоменко. Современная геометрия. Методы и приложения. М.: Наука, 1986.

[26] В. Б. Берестецкий, Е. М. Лифииц, Л. П. Питаевский. Квантовая электродинамика. М.: Наука, 1990.

[27] А. И. Ахиезер, В. Б. Берестецкий. Квантовая электродинамика. М.: Наука, 1981.

[28] L. H. Thomas. Nature. 1926. V. 117. P. 514.

[29] Y. Aharonov, J. Anandan. Phys. Rev. Lett. 1987. V. 58. P. 1593.

Поступила в редакцию 7.VII.2004 г., после доработки 29.XI.2004 г. 